\title{
COMPARISON OF THE IMPULSE AND M-SEQUENCE UWB RADAR SYSTEMS FOR THE ESTIMATION OF THE WALL THICKNESS
}

\author{
Miroslav REPKO, Ján GAMEC \\ Department of Electronics and Multimedia Communications, Faculty of Electrical Engineering and Informatics, Technical University \\ of Košice, Letná 9, 04200 Košice, Slovakia, E-mail: \{miroslav.repko, jan.gamec\} @ tuke.sk
}

\begin{abstract}
In our laboratory, we developed a simple estimation of the wall thickness by the UWB (Ultra Wide-Band) radar system. The described technique measures the wall thickness in the two steps. The first step is a measurement of the wall relative permittivity. The second step is a reflections localisation and estimation of the wall thickness. The borders of the wall could be detected by the envelope of the received signals. The described technique calculates the signal envelope by the Hilbert transform. Moreover, the relative permittivity of the wall is estimated by the technique developed in our department as well. The effective relative permittivity is calculated from the frequency dependency of relative permittivity. This technique was developed for the impulse radar system. The aim of this article is compare the results from impulse radar system with $M$-sequence radar system which operates in the time domain as well measuring wall relative permittivity and wall thickness estimation.
\end{abstract}

Keywords: permittivity estimation, Impulse radar. M-sequence radar, UWB radar, wall thickness

\section{INTRODUCTION}

Research in the localisation of moving objects behind the obstacle is very important in the security applications. The police or counter-terrorist units need to localise persons behind the obstacle, where we can consider a wall as an obstacle. In the scenarios inside the building without the camera monitoring system, the usage of the radar system is an appropriate option. The big advantage of the radar system is the system does not have to be installed before some occasion occurs [1] [2] [3]. The radar system has to perform the measurement in the reflection mode from the single side of the wall, because of the possible application.

For that reason, the radar system requires the data about the thickness and permittivity of the wall. The desired data are used in the process of the person localisation in the part of the wall effect compensation. The compensation of the wall effect tells about the lower velocity of the transmitted EM (Electro-Magnetic) wave inside the wall. The system of estimation of the wall thickness and permittivity increase the accuracy of the person localisation [4].

For the application in the security area, the radar system, as well as used method, need to fulfil several conditions:

- measurement has to be performed from the single side of the wall (reflection mode),

- measurement has to be no-destructive,

- calculation of the results has to be fast,

- measured system has to be cheap.

In our laboratory, we developed a simple technique used by the UWB radar for measurement a wall thickness and its permittivity [5]. The method performs a measurement with the same devices as in process in the localisation of moving persons. This is making a cheap solution for measurement. The presented method use only reflected signals for the calculation of the wall thickness and its permittivity. The UWB radar uses the antennas for the localisation of moving persons as well as for the measurement of the relative permittivity and thickness of the wall. It means that the measurement is non-destructive. The developed method is very simple that means the calculation of results is fast. The described method is possible to use by the impulse radar system as well as the M-sequence radar system [6].

The method performs measurement in two steps. The first step is the measurement of the relative permittivity of the wall by the reflection coefficient where the halfinfinite model of the wall is used. The second step is the estimation of the wall thickness from the envelope of the reflected EM wave from the measured wall.

There exist another technique for the estimation of the wall thickness [7]. However, the estimation of the wall permittivity is performed in insufficient accuracy.

The described method was developed for the impulse radar system. The method requires the radar system operates in the time domain. Another type of the radar system operating in the time domain is M-sequence. This article compares the results of this radar system in the described application. The main difference in the radar system is the impulse radar system transmits the short impulse instead of the M-sequence radar system which is continuously transmitting M-sequence signal. On the other site, the mean value of the M-sequence signal is zero instead of the impulse signal.

The results of the estimated wall thickness are at the end compared to the result from the direct measurement performed by the laser measuring tool.

\section{METHOD FOR ESTIMATION OF RELATIVE PERMITTIVITY}

Permittivity, also called dielectric constant, describes the interaction of the material and the electric field. It is complex frequency dependent function which is unique for every material. In the practise, the relative permittivity is used instead of the full form. The relative permittivity $\varepsilon_{r}$ 
can be defined as:

$\varepsilon_{r}=\frac{\varepsilon}{\varepsilon_{0}}=\varepsilon_{r}^{\prime}-j \varepsilon_{r}^{\prime \prime}=\varepsilon_{r}^{\prime}\left(1-j \tan \delta_{\varepsilon}\right)$,

where $\varepsilon$ is absolute permittivity of the wall and $\varepsilon_{0}$ is permittivity of the vacuum. $\varepsilon_{r}^{\prime}$ and $\varepsilon_{r}^{\prime \prime}$ are real and imaginary parts of relative permittivity and $\tan \delta_{\varepsilon}$ represents a dissipation factor.

The complex permeability $\mu$ which describe a interaction of magnetic part of EM field and material can be defines similarly [8].

Estimation of the wall parameters is sufficient because of the application. The precise complex long measurement does not have to be performed. It is needful to notice the simplification where the measurements are performed at the wall which can be considered as dielectric material. For the dielectric material, we can consider $\mu_{r}^{\prime}=1$ and $\tan \delta_{\mu}=0$. Moreover, if we consider that the dissipation factor $\tan \delta_{\varepsilon}$ is much smaller than relative permittivity $\varepsilon_{r}^{\prime}$ than we can also consider $\tan \delta_{\varepsilon}=0$.

This method is based on the measurement of the reflection coefficient of the wall $\Gamma$. The reflection coefficient $\Gamma$ represents a sum of elementary reflections and transmissions from the single side of the wall. The problem of the multireflection inside of the wall is possible to show in the following diagram (fig. 1).
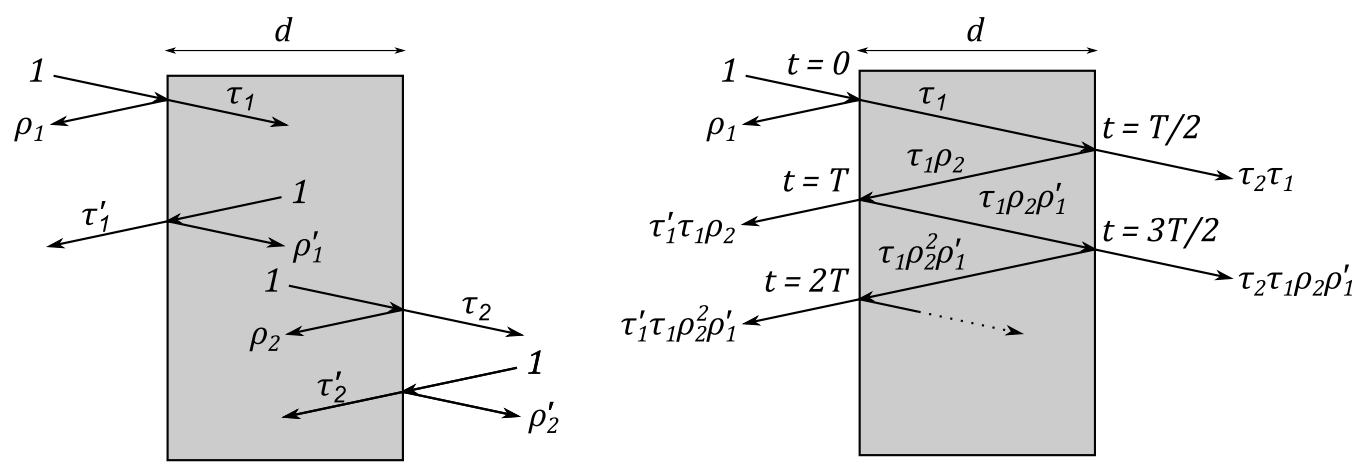

Fig. 1 Diagram of multireflection inside of the wall.

In the fig. 1. $\rho$ represents the elementary reflection coefficient and $\tau$ is a elementary transmission coefficient. The index number represents a number of border and the apostrophe determines the direction of the wave motion. $T$ represents the time period when the transmitted wave appearers again at the incident border of the wall. Then the reflection coefficient is the ratio of the backwards moving participations of the wave and the incident wave. In the case in the fig. 1, where the incident wave is " 1 ", the reflection coefficient $\Gamma$ is possible to calculate by the equation [9]:

$\Gamma(t)=\rho_{1} \delta(t)+\sum_{n=1}^{\infty} \tau_{1} \tau_{1}^{\prime}\left(\rho_{1}^{\prime}\right)^{n-1} \rho_{2}^{n} \delta(t-n T)$.

In the half-infinite model of the wall (thickness of the wall is infinite), the first elementary reflection from the border of the wall $\rho_{1}$ exists only. Then, we can consider that the first elementary reflection $\rho_{1}$ corresponds the reflection coefficient $\Gamma$ for the half-infinite model of the wall, where the reflection from the second border of the wall does not exist. Then we can consider, the reflection coefficient $\Gamma$ equals first elementary reflection $\rho_{1}$ in the scenario where the reflection from the second side of the wall does not exist (when the half-infinite model is used). The first elementary reflection $\rho_{1}$ can be calculated by the equation:

$\rho_{1}=\frac{Z_{1}-Z_{0}}{Z_{1}+Z_{0}}=\frac{Z-1}{Z+1}=\frac{\sqrt{\frac{\mu_{r}^{\prime}\left(1-j \tan \delta_{\mu}\right)}{\varepsilon_{r}^{\prime}\left(1-j \tan \delta_{\varepsilon}\right)}}-1}{\sqrt{\frac{\mu_{r}^{\prime}\left(1-j \tan \delta_{\mu}\right)}{\varepsilon_{r}^{\prime}\left(1-j \tan \delta_{\varepsilon}\right)}}+1}$ where $Z_{1}$ is characteristic impedance of measured material, $Z_{0}$ is characteristic impedance of the free space (vacuum) and $Z$ is the normalized characteristic impedance of measured material which depends on its permittivity and permeability [9]. The impulse radar or M-sequence radar perform a measurement in the time domain. It is possible to apply a window function on received signals in the time domain. If we can predict places of the first and the second reflection on received signals, then the window function can be applied on received signals to suppress the reflections from the second border of the wall. However, the end of the window function has to be before $t=T$. Then the received signal consists only the data of the first elementary reflection $\rho_{1}$. The higher participations of the equation (2) (all summation) can be cancelled for the calculation of the reflection coefficient $\Gamma$ and halfinfinite model of the wall (where $d \rightarrow \infty$ ) can be used. After application of the window function by the way described above, we can write:

$\Gamma=\frac{\sqrt{\frac{\mu_{r}^{\prime}\left(1-j \tan \delta_{\mu}\right)}{\varepsilon_{r}^{\prime}\left(1-j \tan \delta_{\varepsilon}\right)}}-1}{\sqrt{\frac{\mu_{r}^{\prime}\left(1-j \tan \delta_{\mu}\right)}{\varepsilon_{r}^{\prime}\left(1-j \tan \delta_{\varepsilon}\right)}}+1}$

As noticed above, if the measurement is performed at the dielectric material, then is possible to use a substitution where $\mu_{r}^{\prime}=1$ and $\tan \delta_{\mu}=0$. Moreover, if we consider that the dissipation factor is very small compare to the $\varepsilon_{r}^{\prime}$, then we can write that $\tan \delta_{\varepsilon}=0$ as well. After simplifica- 
tion and derivation, the equation (4) can be written as:

$$
\varepsilon_{r}^{\prime}=\left(\frac{1-|\Gamma|}{1+|\Gamma|}\right)^{2}
$$

\section{ESTIMATION OF THE WALL THICKNESS}

The second part of the measurement is the estimation of the wall thickness. This part requires the envelope of the received signal in the time domain, which was reflected from the wall. The envelope is calculated by the Hilbert transform. Received signal is transformed to the analytic signal by adding its Hilbert composition. This analytic signal is complex and in the same domain as original signal (in this case in the time domain). The magnitude of the analytic signal can be considered as the envelope of the original signal.

The main feature of reflections from the wall boundaries in the envelope of the received signal are significant peaks. The position of these peaks in the time corresponds to the boundaries of the wall. This information is also helpful in the first part of estimation when the window function is applied. At first, we need to know the time when electromagnetic waves reached the borders of the wall. It is possible to recognize when the signal was received by the antenna from the envelope of the received signal. Time $t_{1}$, belonging to the reflection from the first border of the wall, can be marked as a place of the first border of the wall. For the explanation, we can consider $t_{1}=0$. Then the time of arrival of the reflection from the first border is 0 and just reflection from the second border of the wall is interesting because it carries the information about the wall thickness. Time $t_{2}$, representing the reflection from the second border of the wall, belongs to the time when the second reflection arrival to the place of the first border. Then we can say that $\Delta t=t_{2}-t_{1}$ carries the information about the time necessary for the EM wave to travel from the first border of the wall to the second and back. In other words, the EM wave travels a thickness of the wall $d$ twice. In the fig. 1 time $\Delta t=T$.

As well known, the velocity of the wave inside the wall compared to the velocity of the light is smaller by the square root of the wall relative permittivity. After that consideration the thickness of the wall $d$ can be calculated by the equation:

$d=\frac{c \Delta t}{2 \sqrt{\varepsilon_{r}^{\prime}}}$

\section{MEASUREMENT SYSTEM}

In the following figure, it is possible to see the diagram of the measurement.

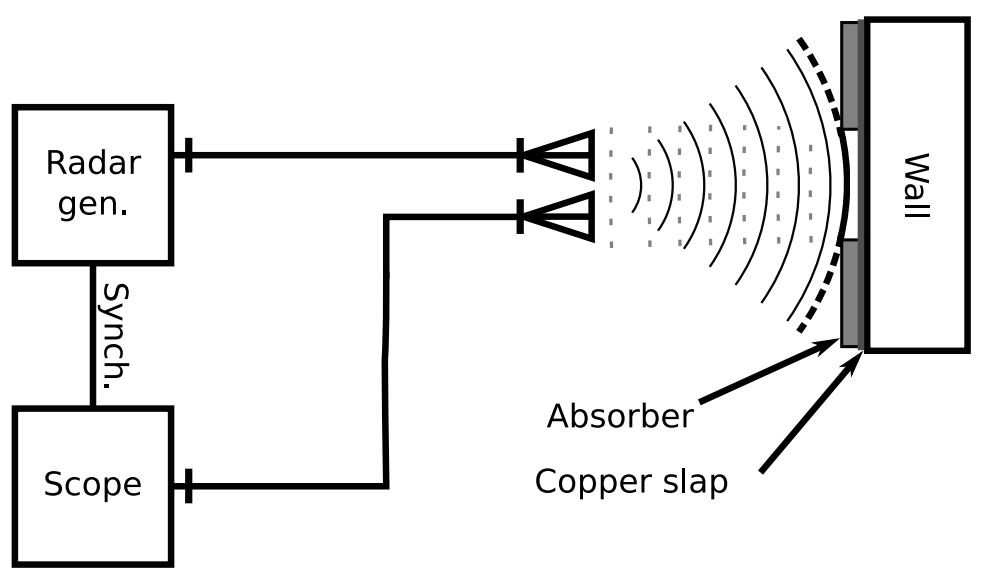

Fig. 2 Diagram of the measurement.

Radar generator and the Scope are parts of the radar system. We perform several measurements by impulse radar system as well as M-sequence radar system. However, we present just demonstration measurements in this paper. The presented measurement was performed by the impulse radar Geozondas (GZ1117DN-50, SD-10806) or M-sequence radar (Ilmsens SH-3100). Double ridged waveguide horn antennas DRH10 (from RF-spin company) are connected via $50 \Omega$ lines to the radar system. The copper slab is a part of the GRL calibration process. Absorbers (ECOSORB AN-77) are used for the attenuation of the incident wave in the places on the wall border with the significant curvature of the wave-front compare to the wall. Usage of the absorber can be prevented by the antennas which wave-front is parallel to the surface of the wall. The measurement was performed in our laboratory on the $0.17 m$ thick brick wall.

GRL (Gated Reflected Line) calibration process is a free space calibration process which is a member of the TRL (Thrue Reflect Line) calibration family.

The reflection coefficient $\Gamma$ can be calculated as the ratio of the reflected and incident wave. For the calculation of $\Gamma$, the method requires the reflection from infinite impedance in the place of the wall border. The reflection from infinite impedance behaves as the incident wave. The calibration process helps to find out the shape of the incident wave. The copper slab behaves as the short circuit for the EM wave. The reflected wave from short is phase 
shifted by $\pi$ rad compare to the reflected wave from infinite impedance.

Presented equations are valid just in the case when the wave-front and the border of the wall are parallel. For that reason, the absorbers were used. Eight absorbers were placed to the square shape $(3 \times 3)$ with the hole in the middle. The antennas were situated in the distance $1.2 \mathrm{~m}$ from the wall. In the fig. 2, the solid narrow lines represent the incident wave. The thick part of the wave-front of the incident wave can be considered as parallel to the wall border. The rest of the incident wave (dashed thick line) is attenuated by the used absorbers. The grey dashed lines represent the reflected wave.

The measurement, including the calibration process, consists of three measurements:

- measurement of the wall,

- measurement of the copper slab (calibration process, reflection behaves as the reflection from short reference),

- measurement of the free space (represents the influence of the measured system. Can be considered as the measurement of the perfect match in the GLR calibration process).
After that consideration, the equation (5) can be written as:

$\varepsilon_{r}^{\prime}=\left(\frac{1+\left|\frac{\mathscr{F}\left(\left(h_{w}(t)-h_{f}(t)\right) \cdot w(t)\right.}{\mathscr{F}\left(\left(h_{f}(t)-h_{s}(t)\right) \cdot w(t)\right.}\right|}{1-\left|\frac{\mathscr{F}\left(h_{w}(t)-h_{f}(t)\right) \cdot w(t)}{\mathscr{F}\left(\left(h_{f}(t)-h_{s}(t)\right) \cdot w(t)\right.}\right|}\right)^{2}$

where $h_{w}(t), h_{s}(t)$ and $h_{f}(t)$ are received signals from the wall, short reference and free space respectively. $w(t)$ represents used window function. $\mathscr{F}(\cdot)$ represents a Fourier transform. This equation have exchanged signs $(+/-)$ compare to equation (5), because phase shift by $\pi \mathrm{rad}$. It should be noticed, the multiplication/deviation in the equation (7) are in real the dot multiplication/deviation were the sample is multiplied/divided by the corresponding sample only.

\section{MEASUREMENT}

As noticed above, we performed three measurements for every radar. Results after subtraction of the device influence are shown in the following figures (fig. (3) and fig. (4)).

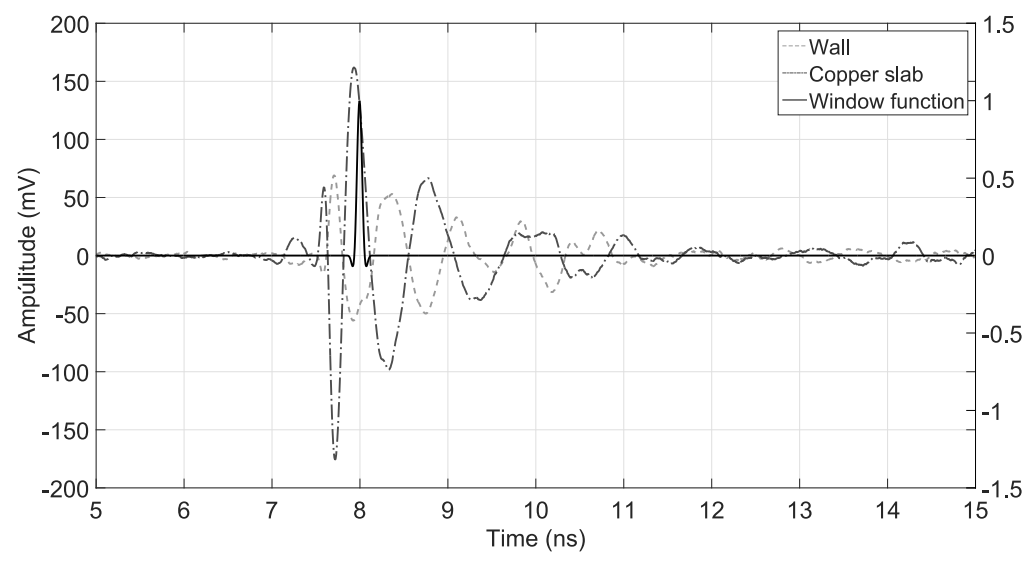

Fig. 3 Received signals and used window function from impulse radar.

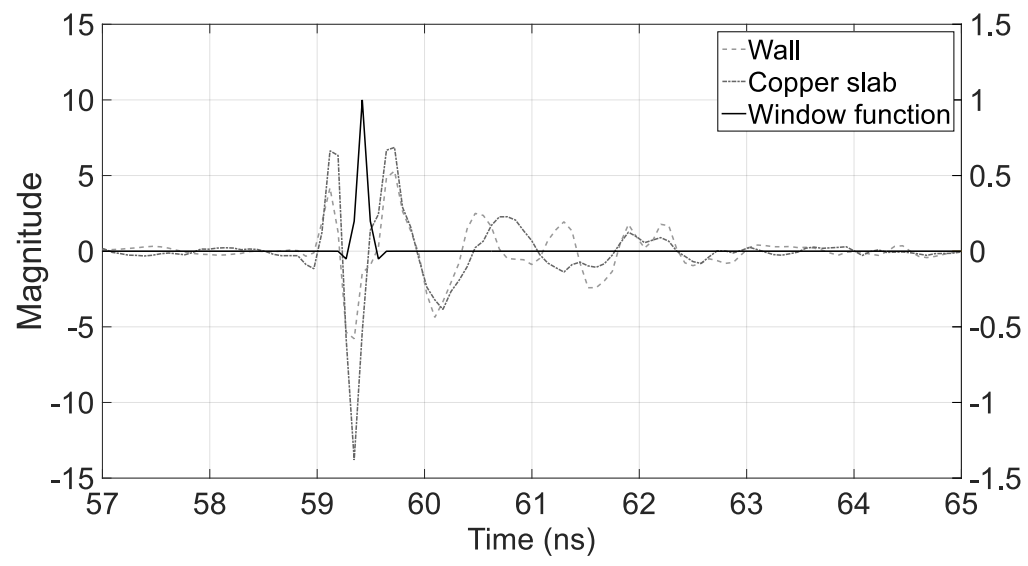

Fig. 4 Received signals and used window function from M-Sequence radar. 
The figures (fig. (3) and fig. (4)) show received signals by the antenna. The dashed bright grey line represents a reflection from the wall and the dot dashed dark grey line represents a reflection from the copper slab. The dot dashed dark grey signal can be considered as an incident wave by the conditions noticed above. Moreover, the figures show a length and a position of the used Flat-top window functions. The window functions are positioned in the highest peak of the signals, where the most of the power of the signals is contained. The length of the window function for the impulse radar system was set by its frequency properties. The window function for M-Sequence radar from the point of view of the frequency properties should be narrower, but than just a small number of samples in the result. There has to be a fulfilled condition that the window functions must end before the reflection from the second side of the wall occurs. That cause the frequency narrower measurement of the relative permittivity. The results of relative permittivity, calculated from the equation (7) of the received data in the fig. 3 and fig. 4, are shown in the following figure (fig. (5)).

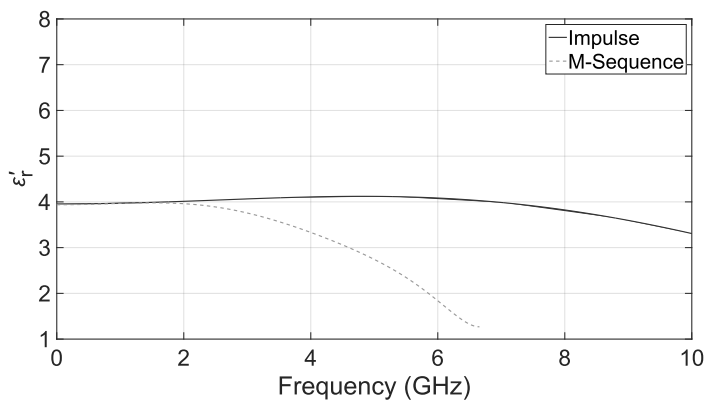

Fig. 5 Measured relative permittivity of the wall.

The frequency properties of used antennas and antialiasing filter cause the valid results in the fig. 5]are in the range $0.6-6 \mathrm{GHz}$. However the radars have a different sampling frequencies and that cause usage of different window functions. For that reason, the result of relative permittivity in the range $0.6-2 \mathrm{GHz}$ are considered only, where the difference between them is small. The effective relative permittivity can be calculated as the geometric mean of the data in the frequency range. For the data in the fig. 5, the calculated relative effective permittivity for the impulse radar system is $\varepsilon_{r_{e f f, i m}}=3.9837$ and for the M-Sequence radar system $\varepsilon_{r_{e f f, m s}}=3.9697$.

The envelopes of reflected signals from the wall are shown in the following figures.

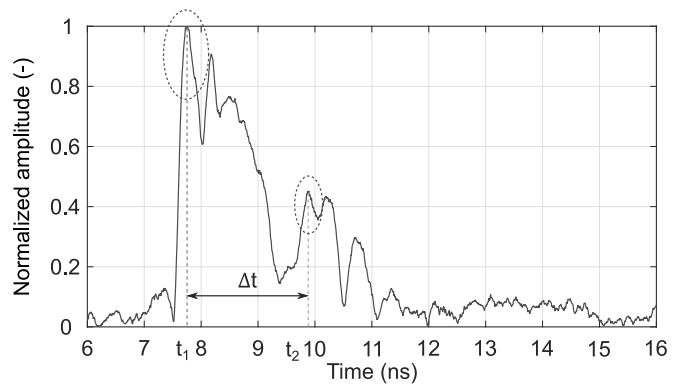

Fig. 6 Envelope of the received signal for the impulse radar.

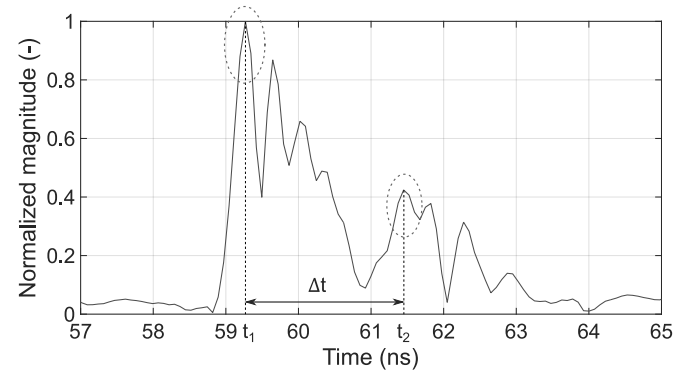

Fig. 7 Envelope of the received signal for the M-sequence radar.

In the fig. 6 and fig. 7, it is possible to clearly see the first and the second border of the wall at position of $t_{1}$ and $t_{2}$ at the peaks of the signal envelope after the Hilbert transform. For the impulse radar system, the values are $t_{1}=7.723 n s$ and $t_{2}=9.892 \mathrm{~ns}$. Than $\Delta t_{i m}=2.169 n s$. For the M-Sequence radar the times are $t_{1}=59.268 n s$ and $t_{2}=61.4483 n s$. which means that $\Delta t_{m s}=2.1785 n s$. Delta times can be used with calculated $\varepsilon_{r_{e f f}}$ in the equation 6. The estimated thicknesses of the wall are $d_{e s, i m}=162.9 \mathrm{~mm}$ and $d_{e s, m s}=163.9 \mathrm{~mm}$.

We performed another verification measurement of the wall thickness by the laser measure (Leica Disto D8). The measured thickness of the wall by the direct method was $d_{m}=173.2 \mathrm{~mm}$. The difference in the results can be caused the accuracy the laser measure and radar system. However, the method of the estimation of the wall thickness works with sufficient accuracy for localisation of moving objects application.

\section{CONCLUSION}

This paper focuses on the method of the estimating the relative permittivity and wall thickness by the UWB radar systems. The presented method can be used by the impulse radar system as well as the M-sequence radar system because both perform measurements in the time domain. In this paper is presented one demonstration measurement for both radar systems. The accuracy of the demonstration measurements are $\Delta d_{i m}=10.3 \mathrm{~mm}$ for the impulse radar system and $\Delta d_{m s}=9.3 \mathrm{~mm}$ what is for the application in the localisation of moving persons sufficient accuracy. The difference in the $\varepsilon_{r_{e f f}}$ can be caused by the influence of the window functions on the received signals. The source of inaccuracy for $\Delta t$ could be a fact that the signal transmitting from the material with hight characteristic impedance to the material with lower characteristic impedance and vice versa changes its phase. For that reason, the borders of the wall does not have to be on the top of the peaks of the envelope bud in the rising edges of the peaks. Also, verification measurement of the wall thickness can be influenced by the accuracy of the laser measure or roughness of the wall. However as was noticed above, both radar systems provide a sufficient results for their usage in the estimation of the wall thickness and relative permittivity. 


\section{ACKNOWLEDGEMENT}

This work was supported by the Slovak Research and Development Agency under the contract No. APVV-15-0692. This work was supported by the Science Grant Agency - project VEGA 1/0772/17.

\section{REFERENCES}

[1] KOCUR, D. - ROVŇÁKOVÁ, J.: Experimental Comparison of Two UWB Radar Systems for the Throughwall Tracking Application, Acta Electrotechnica et Informatica 2, No. 12 (2012) 59-66

[2] NOVÁK, D. - SCHNEIDER, J. - KOCUR, D.: Static Person Detection and localization Based on Their Respiratory Motion Using Various Antenna Types, Acta Electrotechnica et Informatica 16, No. 3 (54-59) 2016

[3] KAŽIMÍR, P. - KOCUR, D.: Simple Method of Uncooperative Human Beings Localisation in 3D Space by UWB Radar, Acta Electrotechnica et Informatica 14, No. 4 (8-12) 2012

[4] ROVŇÁKOVÁ, J. - KOCUR, D.: Compensation of Wall Effect for Through Wall Tracking of Moving Targets, Radioengineering 18, No. 2 (2009) 189-195

[5] REPKO, M. - GAMEC, J.: Simple Permittivity of the Wall Estimation Technique by the UWB Radar System 2017 Progress In Electromagnetics Research Symposium (2017) 1703âĂŞ1708

[6] REPKO, M. - GAMEC, J.: Comparison of Impulse and M-sequence Radar System for Estimation of Relative Permittivity, $19^{\text {th }}$ International Radar Symposium (IRS) (2018) 1âĂ
[7] PROTIVA, P. - MRKVICA, J. - MACHÁČ, J.: Estimation of Wall Parameters from Time-Delay-Only Through-Wall Radar Measurements, IEEE Transactions on Antennas and Propagation 59, No. 11 (2011) $4268-4278$

[8] WHITE, J. F.: Hight Frequency techniques: An Introduction to RF and Microwave Design and Computer Simulation, John Wiley \& Sons (2004)

[9] ORFANIDIS, S. J.: Electronic waves and antennas, Rutgers University, 2016

Received November 09, 2018, accepted March 03, 2019

\section{BIOGRAPHY}

Miroslav Repko was born in 1990. In 2015 he graduated (Ing.) at the department of electronics and multimedia communication of the Faculty of Electrical Engineering and Informatics at Technical University in Košice. Since 2015 he is working as a $\mathrm{PhD}$. student with the department of electronics and multimedia communication. His scientific research is focusing on the measurement of intrinsic parameters of the materials.

Ján Gamec (Assoc. Prof., Ing., Ph.D.), was born in Stul' any, Slovakia in 1960. He graduated from the Technical University in Košice with specialization in Radiotechnics, Summa cum laude in 1985. He reached a Ph.D. degree in radioelectronics at the Technical University, Košice, Slovakia, in 1995. Currently he is an associated professor at the Department of Electronics and Multimedia Communications of the Faculty of Electrical Engineering and Informatics at Technical University in Košice. His main area of scientific research is digital image processing and UWB radar signal processing. 